\title{
Common Carotid Intima-media Thickness in Patients with Non-alcoholic Fatty Liver Disease: A Population-based Case-control Study
}

\author{
Kamran B. Lankarani ${ }^{1}$, Mojtaba Mahmoodi ${ }^{1}$, Mehrzad Lotfi ${ }^{2,3}$, Nima Zamiri ${ }^{1}$, Sayed Taghi Heydari ${ }^{4}$, Fariborz \\ Ghaffarpasand $^{5}$, Mohammad Kazem Fallahzadeh ${ }^{1}$, Meisam Babaeinejad ${ }^{2,3}$, Najmeh Maharlouei ${ }^{1}$, Omid \\ Mirzaee $^{1,6}$, Bita Geramizadeh ${ }^{7,8}$ and Payam Peymani ${ }^{1}$ \\ Health Policy Research Center, Shiraz University of Medical Sciences, Shiraz ${ }^{1}$, Department of Radiology, Namazi Hospital, School of \\ Medicine, Shiraz University of Medical Sciences, Shiraz ${ }^{2}$, Medical Imaging Research Center, Namazi Hospital, Shiraz University of Medical \\ Sciences, Shiraz ${ }^{3}$, Department of Biostatistics, Jahrom University of Medical Sciences, Jahrom ${ }^{4}$, Trauma Research Center, Shiraz University \\ of Medical Sciences, Shiraz ${ }^{5}$, Student Research Committee, Shiraz University of Medical Sciences, Shiraz ${ }^{6}$, Department of Pathology, \\ School of Medicine, Shiraz University of Medical Sciences, Shiraz ${ }^{7}$, Transplant Research Center, Namazi Hospital, Shiraz University \\ of Medical Sciences, Shiraz ${ }^{8}$, Iran
}

Background/Aims: Metabolic syndrome is a well-known risk factor for atherosclerosis. Non-alcoholic fatty liver disease (NAFLD) has features of metabolic syndromes. This study aimed to investigate the association between NAFLD and atherosclerosis. Methods: In a population-based study in southern Iran, asymptomatic adult inhabitants aged more than 20 years were selected through cluster random sampling, and were screened for the presence of fatty liver and common carotid intima-media thickness (CIMT), with abdominal and cervical ultrasonography, respectively. Those with fatty liver were compared to the same number of individuals without fatty liver.

Results: Two hundred and ninety individuals were found to have fatty change on abdominal ultrasonography, and were labeled NAFLD. Compared to normal individuals, NAFLD patients had significantly higher prevalence of increased CIMT (OR, 1.66; $\mathrm{p}<0.001$ ). Those with hypertension (HTN), diabetes mellitus (DM), higher waist circumference (WC) and older ages had significantly higher prevalence of thick CIMT. Through adjusting the effects of different variables, we indicated that NAFLD could be an independent risk factor for thick common carotid intima-media (OR, 1.90; 95\% Cl, 1.17-3.09; $p=0.009$ ). It was also shown that age could be another independent risk factor for thick CIMT.

Conclusions: Individuals with risk factors such as HTN, DM, and high WC are prone to develop atherosclerosis of the carotid artery. The presence of NAFLD should be considered as another probable independent factor contributing to the development of carotid atherosclerosis. (Korean J Gastroenterol 2013;62:344-351)

Key Words: Thickness, intima-media; Artery, common carotid; Liver, fatty

\section{INTRODUCTION}

Nonalcoholic fatty liver disease (NAFLD) encompasses a spectrum of pathologic conditions, ranging from simple steatosis, to nonalcoholic steatohepatitis (NASH) and cirrhosis. ${ }^{1}$ The disease is now the most common cause of elevated liver enzymes worldwide, including developing and developed countries. Approximately $20-30 \%$ of adults in the general population of western countries have NAFLD, ${ }^{2,3}$ and its prevalence increases to $70-90 \%$ among individuals who are obese or have diabetes. ${ }^{4}$ Recent study from Iran (as a developing country) indicated the prevalence of $21.5 \%{ }^{5}$ Regardless of its hepatic sequel, there is an increasing trend of evidence suggesting that NAFLD is the missing part of metabolic syn-

Received April 24, 2013. Revised October 31, 2013. Accepted November 18, 2013.

(C) This is an open access article distributed under the terms of the Creative Commons Attribution Non-Commercial License (http://creativecommons.org/licenses/ by-nc/3.0) which permits unrestricted non-commercial use, distribution, and reproduction in any medium, provided the original work is properly cited.

Correspondence to: Mojtaba Mahmoodi, Health Policy Research Center, Shiraz School of Medicine, Shiraz University of Medical Sciences, Zand Blvd., Shiraz, Iran. Tel: +98-711-230-9615, Fax: +98-711-230-9615, E-mail: mahmoodimoj@gmail.com

Financial support: This project was financially supported by the Health Policy Research Center, Shiraz University of Medical Sciences, Shiraz, Iran. Conflict of interest: None. 
drome (i.e. a sixth criterion). From this point of view, NAFLD strongly relates with diabetes mellitus, hypertension, and obesity, ${ }^{6}$ and consequently, with increased risk for cardiovascular events. The atherogenic effect of metabolic syndrome was previously established, as well as its components; but the role of NAFLD in this regard is still being debated. There are evidences of increased rate of cardiovascular mortality in patients with NAFLD and/or cirrhosis, but the role of NAFLD as the main etiology, or as just a co-morbid disease has remained unclear. ${ }^{7,8}$ Many published studies focusing on this issue were done in symptomatic patients with NAFLD, or on patients in clinical follow up. Few studies, if any, evaluated this relation in a population-based setting, with the participation of asymptomatic individuals.

Intima-media thickness (IMT) of the common carotid artery is a reliable predictor of atherosclerosis. Increased common carotid intima-media thickness (CIMT) has been considered as a marker of atherosclerosis and cardiovascular disease. This study was conducted to find the relation of NAFLD and atherosclerosis as the main predictor of future cardiovascular disease, in a population-based setting, through comparison of the IMT of carotid artery and other important cardiovascular risk factors, between patients with NAFLD and controls.

\section{SUBJECTS AND METHODS}

1. Study population

This study was an extension of our previous study on the prevalence of fatty liver disease in the general population in Shiraz, southern Iran, which has recently been published. ${ }^{5}$ Based on ultrasonograhic finding, as described in the following parts, individuals with fatty liver disease were identified.

Flow chart of the study is demonstrated in Fig. 1. Among 819 individuals who agreed to participate in the study, 342 had evidence of fatty liver disease on ultrasonography in any form, from mild to severe (ultrasonographic criteria are described in the "study protocol" part).

We excluded patients with positive or suspicious results for HBsAg, anti-HCV and HIV. We also excluded subjects who had any history of liver disease, had suspicious hepatic-related signs and symptoms during the physical exam or after history taking, had a history of pregnancy in the previous year, had a history of weight loss or weight gain in recent years, or who had had any kind of major organ failure. Exclusion of individuals reporting alcohol consumption was ensured, by interviewing the participants at two different times. We excluded pregnant women, or those who had delivered within the past six months. Due to different genetic and environmental background, which could have affected our results, non-Iranian residents were also excluded.

Fifty-two of these individuals were excluded, based on the

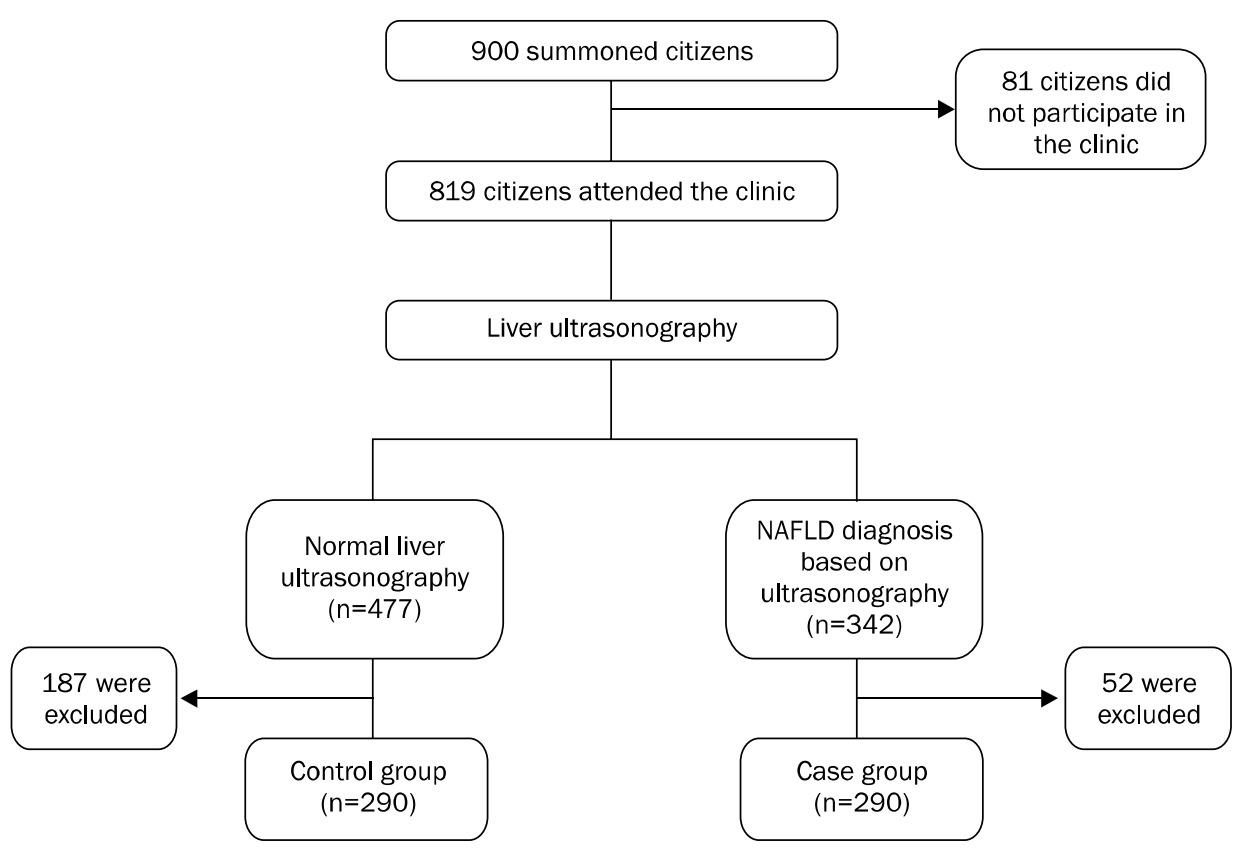

Fig. 1. A flow chart of the study. 
above-mentioned exclusion criteria. One age matched control group were selected from those who attended our clinic and had normal liver ultrasonography $(n=477)$. Age matching was based on ten-year periods. The above-mentioned exclusion criteria were also applied for selecting the control group.

The approvals of the Institutional Review Board, as well as of the Ethics Committee of Shiraz University of Medical Sciences, were obtained prior to the start of the study (No. HP-12-89). All participants gave their written informed consent.

\section{Study protocol}

All the individuals were invited to come to the Fatty Liver Special Clinic, Health Policy Research Center, Shiraz University of Medical Sciences (Shiraz, Iran) for a comprehensive medical evaluation, including physical examination, and complete multi-disciplinary history taking (by physicians and nurses), and anthropometric assessments (by trained nurses). Venous blood samples were also taken, for related laboratory analysis.

Diabetes mellitus was defined according to the American Diabetes Association criteria, based on an fasting blood sug$\operatorname{ar}$ (FBS) $>126 \mathrm{mg} / \mathrm{dL} .{ }^{9}$ Hypertension was defined according to the Seventh Report of the Joint National Committee on Prevention, Detection, Evaluation and Treatment of High Blood Pressure (JNC 7). ${ }^{10}$ Afterwards, liver ultrasonography and common carotid artery ultrasonography were performed for all participants.
The National Cholesterol Education Program - Adult Treatment Panel III (ATP III) published criteria ${ }^{11}$ were applied for the diagnosis of metabolic syndrome in our population, which defined metabolic syndrome as positivity for three or more of the following criteria: 1) FBS $>110 \mathrm{mg} / \mathrm{dL}$, or current use of hypoglycemic agents; 2) central obesity (waist circumference $>102 \mathrm{~cm}$ in men, and $>88 \mathrm{~cm}$ in women); 3) arterial blood pressure $>130 / 85 \mathrm{mmHg}$, or current use of antihypertensive medications; 4) triglyceride levels $>150 \mathrm{mg} / \mathrm{dL}$, or current use of fibrates; 5) HDL cholesterol $<40 \mathrm{mg} / \mathrm{dL}$ in men, and $<50 \mathrm{mg} / \mathrm{dL}$ in women.

\section{Liver ultrasonography}

Participants underwent abdominal ultrasonography according to a standardized protocol, taking into consideration that ultrasonography can detect increased liver echogenicity, and confirm the diagnosis of NAFLD, particularly when hepatic fat infiltration surpasses 33\%. ${ }^{12}$ Real-time imaging of the liver was performed for each subject by an experienced radiologist, using a Shimadzu ultrasound machine (Shimadzu Inc., Tokyo, Japan) with a 5-MHz to 7-MHz transducer probe (curvilinear). The radiologist performing the ultrasonography was unaware of the clinical and laboratory results. The ultrasonography diagnostic patterns of fatty liver disease were based on the presence of a 'bright' liver, with stronger echoes in the hepatic parenchyma than in the renal parenchyma, often associated with unusually fine liver echotexture and vessel blurring, in the absence of findings suggestive of other chronic liver diseases.
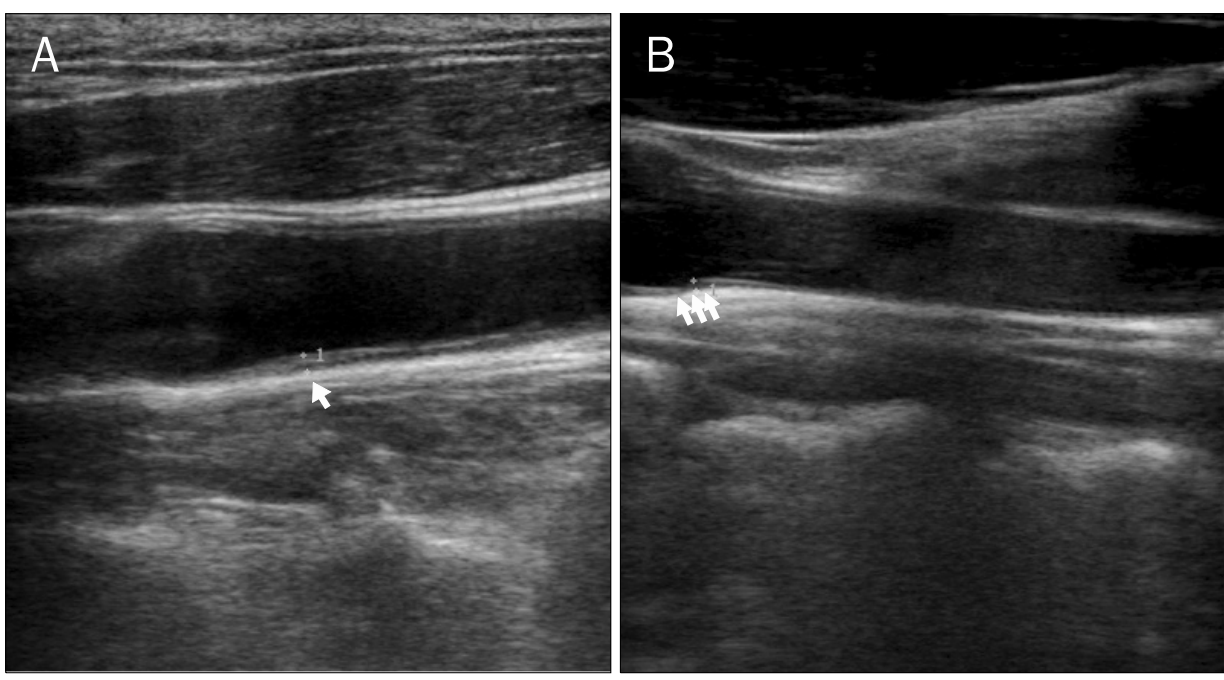

Fig. 2. Longitudinal ultrasonographic image of the common carotid artery. Longitudinal ultrasonographic image of the common carotid artery, showing the carotid intima-media thickness in the posterior wall (diameter: $11 \mathrm{~mm}$ $[\mathrm{A}], 7 \mathrm{~mm}[\mathrm{~B}])$. 


\section{Carotid artery ultrasonography}

Carotid artery ultrasonography was performed using the same model of ultrasonography scanner (Sonoline G50; Seimens AG, Munich, Germany). The protocol involved scanning of both common carotid, as well as internal and external carotid arteries. Subjects were examined by an expert radiologist, following a standardized protocol. Briefly, IMT was assessed in the posterior wall of the artery as the distance between the leading edge of the first and second echogenic lines (Fig. 2). All of the scans were recorded. The best-quality end diastolic image was captured in a longitudinal axis view that showed the bifurcation, and was analyzed off-line. From this image, three measurements of the common carotid artery were performed at a distance of $0.5 \mathrm{~cm}, 1 \mathrm{~cm}$, and $2 \mathrm{~cm}$ below the bifurcation of the common carotid, and the average measurement was used for further assessments. The cut-off value for the CIMT was set at $0.8 \mathrm{~mm}$ (CIMT $\geq 0.8 \mathrm{~mm}$ ), in order to avoid missing any patients. ${ }^{13}$ All measurements were carried out by a single radiologist on previously recorded scans. At the time of reading, he was unaware of the patients' identities, and clinical and laboratory characteristics.

\section{Statistical analysis}

Statistical analyses were performed using the SPSS software, version 17.0 (SPSS Inc., Chicago, IL, USA). Results are reported as the mean $\pm S D$ for continuous variables, and as number (\%) for categorical variables. For the comparison between patient and control data, student's t-test were used for quantitative variables and the chi-square test was used to determine the statistical significance of differences in qualitative variables. Multiple logistic regression analysis (adjusted OR with multiple logistic regression) was performed for the adjustment of different risk factors and variables associated with the development of thick CIMT.

\section{RESULTS}

1. Comparison of clinical characteristics between NAFLD and the control group

Table 1 shows the demographical, clinical and laboratory characteristics of patients and controls. The 290 patients with NAFLD and the same number of controls were matched in term of age. In the NAFLD group, 160 (55.2\%) participants were females, while in the controls, 174 (60\%) individuals were females $(p=0.27)$. The mean age of patients with NAFLD was $46.4 \pm 11.9$ years, with a value of $45.4 \pm 12.4$ years in the controls $(p=0.90)$. Patients with NAFLD had a higher blood pressure (both systolic and diastolic) than the controls, with statistical significance $(p<0.001)$. Mean FBS was significantly higher in patients with NAFLD, than in the controls (101.8 \pm 32.9 vs. $86.3 \pm 13.5 \mathrm{mg} / \mathrm{dL}, \mathrm{p}<0.001)$. Levels of liver aminotransferases were higher in patients with NAFLD, than in the controls $(p<0.001)$. We also noticed that the level

Table 1. Clinical, Anthropometric and Laboratory Findings of Patients with NAFLD and Control Group (Ultrasonographic Normal Liver Group)

\begin{tabular}{|c|c|c|c|}
\hline & $\begin{array}{c}\text { Ultrasonogra- } \\
\text { phic NAFLD } \\
\text { group } \\
(n=290)\end{array}$ & $\begin{array}{l}\text { Ultrasonogra- } \\
\text { phic normal } \\
\text { liver group } \\
\quad(n=290)\end{array}$ & p-value \\
\hline Hypertension & $82(28.3)$ & $54(18.6)$ & $<0.001$ \\
\hline Diabtes mellitus & $97(33.4)$ & 34 (11.7) & $<0.001$ \\
\hline BMI & $28.6 \pm 4.2$ & $25 \pm 3.8$ & $<0.001$ \\
\hline Normal $(<25)$ & $51(17.6)$ & $145(50.0)$ & $<0.001$ \\
\hline Overweight $(\geq 25,<30)$ & $142(49.0)$ & $110(37.9)$ & \\
\hline Obese $(\geq 30)$ & 97 (33.4) & $35(12.1)$ & \\
\hline Waist circumference (cm) & $95.2 \pm 10.4$ & $86.9 \pm 9.0$ & $<0.001$ \\
\hline $\begin{array}{l}\text { Waist/hip circumference } \\
(\mathrm{cm})\end{array}$ & $0.889 \pm 0.061$ & $0.861 \pm 0.063$ & $<0.001$ \\
\hline $\begin{array}{l}\text { Systolic blood pressure } \\
(\mathrm{mmHg})\end{array}$ & $119.1 \pm 15.9$ & $113.8 \pm 14.2$ & $<0.001$ \\
\hline $\begin{array}{l}\text { Diastolic blood pressure } \\
(\mathrm{mmHg})\end{array}$ & $78 \pm 9.5$ & $74.7 \pm 9.2$ & $<0.001$ \\
\hline Metabolic syndrome & $91(31.4)$ & $39(13.4)$ & $<0.001$ \\
\hline $\begin{array}{l}\text { Fasting blood sugar } \\
(\mathrm{mg} / \mathrm{dL})\end{array}$ & $101.8 \pm 32.9$ & $86.3 \pm 13.5$ & $<0.001$ \\
\hline AST (IU/L) & $26.9 \pm 11.5$ & $23.7 \pm 10.1$ & $<0.001$ \\
\hline ALT (IU/L) & $31.1 \pm 21.1$ & $21.0 \pm 11.0$ & $<0.001$ \\
\hline GGT (IU/L) & $37.4 \pm 27.4$ & $31.8 \pm 31.6$ & $<0.021$ \\
\hline ALP (IU/L) & $208.1 \pm 66.4$ & $194.3 \pm 71.9$ & 0.018 \\
\hline Total cholesterol (mg/dL) & $198.9 \pm 42.1$ & $190.1 \pm 40.5$ & 0.011 \\
\hline LDL (mg/dL) & $112.4 \pm 35.4$ & $108.5 \pm 33.4$ & 0.176 \\
\hline $\mathrm{HDL}(\mathrm{mg} / \mathrm{dL})$ & $49.4 \pm 10.3$ & $51.0 \pm 11.3$ & 0.075 \\
\hline Triglyceride (mg/dL) & $167.8 \pm 84.1$ & $127.2 \pm 60.7$ & $<0.001$ \\
\hline Uric acid (mg/dL) & $4.8 \pm 1.5$ & $4.3 \pm 1.3$ & $<0.001$ \\
\hline Serum ferritin $(\mathrm{ng} / \mathrm{mL})$ & $114.1 \pm 106.2$ & $88.2 \pm 92.5$ & 0.002 \\
\hline ANA (IU/L) & $3.2 \pm 12.8$ & $2.8 \pm 3.4$ & 0.70 \\
\hline CIMT (mm) & & & 0.009 \\
\hline$<0.8$ & 197 (67.9) & 226 (77.9) & \\
\hline$\geq 0.8$ & 93 (32.1) & 64 (22.1) & \\
\hline
\end{tabular}

Values are presented as the mean \pm SD or $n(\%)$.

For the comparison between patient and control data, student's t-test were used for quantitative variables and the chi-square test was used to determine the statistical significance of differences in qualitative variables. p-value less than 0.05 is considered statistically significant.

NAFLD, nonalcoholic fatty liver disease; CIMT, common carotid intima-media thickness. 
Table 2. Comparison between CIMT and Metabolic Syndromerelated Factors

\begin{tabular}{lccr}
\hline & CIMT & CIMT & \\
& $\begin{array}{c}20.8 \mathrm{~mm} \\
(\mathrm{n}=157)\end{array}$ & $\begin{array}{c}<0.8 \mathrm{~mm} \\
(\mathrm{n}=423)\end{array}$ & p-value \\
& $54.4 \pm 11.4$ & $42.8 \pm 10.8$ & $<0.001$ \\
\hline Age (yr) & $58(36.9)$ & $78(18.4)$ & $<0.001$ \\
Hypertension & $52(33.1)$ & $79(18.7)$ & $<0.001$ \\
Diabetes mellitus & $153.7 \pm 79.7$ & $145.1 \pm 74.5$ & 0.251 \\
Triglyceride (mg/dL) & $50.7 \pm 9.9$ & $50.1 \pm 11.2$ & 0.506 \\
HDL (mg/dL) & $93.3 \pm 9.6$ & $90.3 \pm 10.9$ & 0.002 \\
Waist circumference & & & \\
$\quad$ (cm) & $93(59.2)$ & $197(46.6)$ & 0.007 \\
NAFLD & $110.3 \pm 107.7$ & $98 \pm 97.5$ & 0.222 \\
Serum ferritin & & & \\
$\quad(\mathrm{ng} / \mathrm{mL})$ & & & \\
\hline
\end{tabular}

Values are presented as the mean \pm SD or $\mathrm{n}(\%)$.

For the comparison between those with thick CIMT and subjects with normal CIMT, student's t-test were used for quantitative variables, and the chi-square test was used to determine the statistical significance of differences in qualitative variables. p-value less than 0.05 is considered statistically significant. CIMT, common carotid intima-media thickness; NAFLD, nonalcoholicfatty liver disease.

of serum ferritin was significantly higher in patients with NAFLD, than in the controls $(p=0.002)$. Those with NAFLD had a significantly higher prevalence of increased CIMT, in comparison with normal individuals (OR, 1.66; 95\% Cl, 1.15-2.41; $p<0.001)$. We also observed that patients with NAFLD had a higher prevalence of metabolic syndrome (OR, 2.94; $95 \% \mathrm{Cl}, 1.93-4.47 ; \mathrm{p}<0.001$ ), than the controls. However, in the NAFLD group, no correlation was found between the presence of metabolic syndrome and the higher occurrence of increased CIMT (38.5\% in the NAFLD patients with metabolic syndrome, in comparison with $29.1 \%$ in the NAFLD patients without metabolic syndrome, $p=0.136$ ).

\section{Association between CIMT and metabolic syndrome}

A comparison between CIMT and metabolic syndromerelated risk factors is depicted in Table 2 . The mean age of subjects with CIMT equal to or greater than $0.8 \mathrm{~mm}$ was $54.4 \pm 11.4$ years, with a value of $42.8 \pm 10.8$ years in those with CIMT less than $0.8 \mathrm{~mm}(\mathrm{p}<0.001)$. Subjects with thick CIMT had a higher prevalence of HTN, in comparison to those with normal CIMT (36.9\% vs. 18.4\%, $p<0.001)$. This is also true for those with NAFLD, in contrast to subjects without NAFLD (59.2 vs. 46.6, $p=0.007)$.

Individuals with thick CIMT had a significantly higher waist circumference, than those with normal CIMT $(93.3 \pm 9.6$ vs.
Table 3. Multivariate Analysis for the Risk Factors of CIMT $\geq 0.8$ $\mathrm{mm}^{\mathrm{a}}$

\begin{tabular}{|c|c|c|c|}
\hline Variable & p-value & $\begin{array}{c}\text { OR } \\
(\mathrm{CIMT} \geq 0.8 \mathrm{~mm} / \\
<0.8 \mathrm{~mm})\end{array}$ & $95 \% \mathrm{Cl}$ \\
\hline \multicolumn{4}{|l|}{ NAFLD } \\
\hline No & & 1 & - \\
\hline Yes & 0.009 & 1.91 & $1.17-3.10$ \\
\hline \multicolumn{4}{|l|}{ Hypertension } \\
\hline No & & 1 & - \\
\hline Yes & 0.238 & 1.34 & $0.82-2.19$ \\
\hline \multicolumn{4}{|l|}{ Diabetes mellitus } \\
\hline No & & 1 & - \\
\hline Yes & 0.611 & 1.14 & $0.68-1.89$ \\
\hline \multicolumn{4}{|c|}{ Waist circumference ${ }^{b}$} \\
\hline Normal & & 1 & - \\
\hline High & 0.054 & 0.62 & $0.38-1.01$ \\
\hline \multicolumn{4}{|l|}{ Age (yr) } \\
\hline $18-29$ & & 1 & - \\
\hline $30-39$ & 0.166 & 0.27 & $0.04-1.710$ \\
\hline $40-49$ & 0.048 & 3.50 & $1.01-12.18$ \\
\hline $50-59$ & $<0.001$ & 10.83 & $3.07-38.20$ \\
\hline$\geq 60$ & $<0.001$ & 22.76 & $6.08-85.21$ \\
\hline
\end{tabular}

CIMT, common carotid intima-media thickness; NAFLD, nonalcoholic fatty liver disease.

${ }^{a}$ Adjusted OR with multiple logistic regression.

${ }^{\mathrm{b}}$ Waist circumference $>102 \mathrm{~cm}$ in men and $>88 \mathrm{~cm}$ in women was considered high.

$90.3 \pm 10.9 \mathrm{~cm}, \mathrm{p}=0.002)$.

3. Multivariate analysis of risk factors for CIMT $\geq 0.8$ $\mathrm{mm}$

Table 3 indicates the results of the multiple logistic regression analysis (with adjusted OR) of five criteria of metabolic syndrome (diabetes mellitus, hypertension, triglyceride, HDL, and waist circumference), as well as NAFLD for predicting thick common carotid intima-media. Age and serum ferritin level were also mentioned in our analysis, in order to adjust their impact on the CIMT. The table shows that NAFLD could be an independent risk factor for thick common carotid intima-media (OR, 1.9; 95\% Cl, 1.17-3.09; $p=0.009)$.

Statistical analysis showed that patients between 40 to 49 years old were more prone to develop thick CIMT, independent of the presence of other risk factors for thick CIMT (OR, 3.5; 95\% Cl, 1.01-12.18; $p=0.048$ ). This would be more prominent for patients who were between 50 to 59 years old, as well as those above age 60, for detecting thick CIMT (OR, 10.83; 95\% Cl, 3.07-38.2; $\mathrm{p}<0.001$ and OR, 22.76; 95\% Cl, $6.08-85.21 ; p<0.001$, respectively). 


\section{DISCUSSION}

The prevalence of NAFLD is growing globally, as a result of the increase of type II diabetes mellitus and obesity. ${ }^{14}$ The NAFLD is closely related to insulin resistance, and to the presence of markers of oxidative stress in plasma and endothelial dysfunction. The NAFLD is actually mentioned as the hepatic manifestation of the metabolic syndrome. ${ }^{15}$ The increase in risk of cardiovascular disease in patients with metabolic syndrome is considerable. ${ }^{16}$

The importance of NAFLD is highlighted by new data suggesting that NAFLD can be an independent risk factor for cardiovascular disease. ${ }^{17,18}$ As a matter of fact, the hypothesis that NAFLD is not only a marker of cardiovascular disease, but may also be involved in its pathogenesis, ${ }^{19}$ has started to gain more evidence. This situation is in concordance with the new guidelines for the diagnosis and treatment of patients with NAFLD, which emphasize an early and more accurate diagnosis of this pathologic condition, in order to better define the disease progression from simple fatty changes without inflammation, to NASH, cirrhosis, and end-stage liver disease. $^{20}$

Our population-based case-control study suggests that cardiovascular risk factors, such as increased IMT, occur more frequently among NAFLD patients, when compared to healthy individuals, confirming previous findings in patients' NAFLD. ${ }^{8,18,21}$ Although some of these studies included only NAFLD patients with elevated liver aminotransferases, most surveys stated that NAFLD is associated with increased CIMT, regardless of the rise of liver enzymes plasma levels. ${ }^{21}$ Recent studies concluded that the same process occurs in cerebral arteries, which causes increased cerebrovascular resistance. ${ }^{22}$ This leads to a higher risk of morbidity and mortality related to non-hepatic consequences of NAFLD. ${ }^{23}$ Through adjusting the effects of other variables, such as DM and age, we also indicated that NAFLD could be an independent risk factor for thick common carotid intima-media (OR, 1.91; 95\% Cl, 1.17-3.01; p=0.009). Randomized prospective clinical trials are needed, to sufficiently evaluate the direct and indirect impacts of NAFLD on the atherosclerotic process.

In our study, we observed that the age of 50 years is a suitable cut-off value for predicting $\mathrm{CIMT} \geq 0.8 \mathrm{~mm}$. This finding is applicable in practice, because fatty changes in the liver are usually incidental findings in abdominal ultrasonography, and there is a permanent controversy regarding which patients should undergo further laboratory and paraclinical work-up. Recent surveys support the argument that patients with non-alcoholic fatty changes of liver have a higher death probability due to cardiovascular complications, in comparison to the complications of liver dysfunction. ${ }^{24}$ Therefore, it is crucial to perform cardiovascular evaluations in patients with NAFLD, even if no other cardiovascular risk factor is observed. ${ }^{25}$ Overwhelming evidence suggests that cardiovascular disease determines the outcomes in patients with NAFLD more frequently, and to a greater extent, than the progression of liver disease. ${ }^{24,26,27}$

Serum ferritin was increased in NAFLD patients, in comparison with the controls. The role of ferritin in the human body is to store and deliver iron to the cells, and the levels of ferritin in plasma reflect the total body iron supply. Ferritin is also an acute-phase reactant that may have elevated plasma levels, in the case of infection, inflammation, and other stimuli. ${ }^{28}$ Because there are no proofs at present to advocate for a role of excess iron in the pathogenesis of NAFLD, ${ }^{29}$ it can be hypothesized that a higher ferritin level in these patients could be linked to the presence of an inflammatory background associated with steatosis in hepatocytes, and even necrosis. This concept is supported by our findings, which indicate that the serum ferritin level was strongly correlated with markers of hepatocyte injury (alanine aminotransferase, aspartate aminotransferase and gamma-glutamyl transpeptidase correlated with ferritin levels, $p$ $<0.001)$. Although growing evidence suggests a link between serum ferritin, insulin resistance, and NAFLD, ${ }^{30}$ the increased serum ferritin levels may just be in response to, and along with the atherogenic course undergoing in vessel walls. This can be explained on the basis of a mechanism that states that ferritin genes are up-regulated by inflammatory cytokines, and are prone to induction in the process of plaque formation. $^{31}$

The gold standard for the diagnosis of NAFLD is liver biopsy, but because of ethical reasons, the procedure was not applied in our study. As ultrasonography is the most common and readily available method for the diagnosis of fatty changes in liver, we used it, in addition to excluding known etiologies of liver disease. Studies have proven that the optimal hepatic steatosis threshold for radiologic detection is 33\% of 
liver texture, and this is the range of moderate to severe NAFLD. Therefore, regardless of changes in liver enzymes, abdominal ultrasonography is sensitive enough for the detection of fatty changes in the liver. ${ }^{12}$

Several studies have evaluated the relationship between NAFLD and CIMT, and support the idea of independent associations between NAFLD and atherosclerosis. ${ }^{8,20,32-35}$

One of the important defects of many of these studies is that their cases groups are selected from those who were referred to clinics or private physicians. Consequently, these NAFLD subjects do not actually represent the general population. The causes for referral of these NAFLD patients were the development of signs and symptoms that made either them, or their family physicians, initiate diagnostic workups. On the other hand, in our study, the cases group was randomly selected from the general population. The cases did not complain of any symptoms, and their physical exam and history taking by trained physicians revealed no sign and symptoms, NAFLD being detected by ultrasonography.

In summary, we suggest that NAFLD could be a risk factor for carotid atherosclerosis, independently of its association with the metabolic syndrome. The clinical corollary of our findings suggests that the detection of fatty changes in liver on abdominal ultrasonography should provide an alert on the probability of the presence of multiple underlying cardiovascular risk factors, warranting evaluation and treatment, as cardiovascular atherosclerosis-related morbidity and mortality are elevated by the pathological course of fatty liver disease.

\section{ACKNOWLEDGEMENTS}

We sincerely thank all the subjects who participated in our study, as well as their families. We also express our gratitude to all the staff of the Motahari Polyclinic laboratory for their cooperation.

\section{REFERENCES}

1. Ko JS. Nonalcoholic fatty liver disease. Korean J Gastroenterol 2010;56:6-14.

2. Levene AP, Goldin RD. The epidemiology, pathogenesis and histopathology of fatty liver disease. Histopathology 2012;61:141152.

3. Vernon G, Baranova A, Younossi ZM. Systematic review: the epidemiology and natural history of non-alcoholic fatty liver disease and non-alcoholic steatohepatitis in adults. Aliment Pharmacol Ther 2011;34:274-285.

4. Ahmed MH, Abu EO, Byrne CD. Non-alcoholic fatty liver disease (NAFLD): new challenge for general practitioners and important burden for health authorities? Prim Care Diabetes 2010;4: 129-137.

5. Lankarani KB, Ghaffarpasand F, Mahmoodi M, et al. Non alcoholic fatty liver disease in southern Iran: a population based study. Hepat Mon 2013;13:e9248.

6. Lee SY, Kim SK, Kwon Cl, et al. Clinical characteristics of health screen examinees with nonalcoholic fatty liver and normal liver function test. Korean J Gastroenterol 2008;52:161-170.

7. Margariti E, Deutsch M, Manolakopoulos S, Papatheodoridis GV. Non-alcoholic fatty liver disease may develop in individuals with normal body mass index. Annals of Gastroenterology 2012;25: 45-51.

8. Sookoian S, Pirola CJ. Non-alcoholic fatty liver disease is strongly associated with carotid atherosclerosis: a systematic review. J Hepatol 2008;49:600-607.

9. Basevi V, Di Mario S, Morciano C, Nonino F, Magrini N. Comment on: American Diabetes Association. Standards of medical care in diabetes--2011. Diabetes Care 2011;34(Suppl. 1):S11-S61. Diabetes Care 2011;34:e53, author reply e54.

10. Lenfant C, Chobanian AV, Jones DW, Roccella EJ; Joint National Committee on the Prevention, Detection, Evaluation, and Treatment of High Blood Pressure. Seventh report of the Joint National Committee on the Prevention, Detection, Evaluation, and Treatment of High Blood Pressure (JNC 7): resetting the hypertension sails. Hypertension 2003;41:1178-1179.

11. Grundy SM, Cleeman JI, Daniels SR, et al; American Heart Association; National Heart, Lung, and Blood Institute. Diagnosis and management of the metabolic syndrome: an American Heart Association/National Heart, Lung, and Blood Institute Scientific Statement. Circulation 2005;112:27352752.

12. Saadeh S, Younossi ZM, Remer EM, et al. The utility of radiological imaging in nonalcoholic fatty liver disease. Gastroenterology 2002;123:745-750.

13. Dougherty RS, Brant WE. Vascular ultrasound. In: Brant WE, Helms CA, eds. Fundamentals of diagnostic radiology. 3rd ed. Philadelphia: Lippincott, Williams \& Wilkins, 2007:1027.

14. Yki-Järvinen $\mathrm{H}$. Nutritional modulation of nonalcoholic fatty liver disease and insulin resistance: human data. Curr Opin Clin Nutr Metab Care 2010;13:709-714.

15. Kotronen A, Westerbacka J, Bergholm R, Pietiläinen $\mathrm{KH}$, Yki-Järvinen $\mathrm{H}$. Liver fat in the metabolic syndrome. J Clin Endocrinol Metab 2007;92:3490-3497.

16. Musso G, Gambino R, Bo S, et al. Should nonalcoholic fatty liver disease be included in the definition of metabolic syndrome? A cross-sectional comparison with Adult Treatment Panel III criteria in nonobese nondiabetic subjects. Diabetes Care 2008; 31:562-568.

17. Targher G, Day CP, Bonora E. Risk of cardiovascular disease in patients with nonalcoholic fatty liver disease. N Engl J Med 2010; 363:1341-1350.

18. Targher G, Bertolini L, Rodella S, et al. Nonalcoholic fatty liver dis- 
ease is independently associated with an increased incidence of cardiovascular events in type 2 diabetic patients. Diabetes Care 2007;30:2119-2121.

19. Maurantonio M, Ballestri S, Odoardi MR, Lonardo A, Loria P. Treatment of atherogenic liver based on the pathogenesis of nonalcoholic fatty liver disease: a novel approach to reduce cardiovascular risk? Arch Med Res 2011;42:337-353.

20. Loria P, Adinolfi LE, Bellentani S, et al; NAFLD Expert Committee of the Associazione Italiana per lo studio del Fegato. Practice guidelines for the diagnosis and management of nonalcoholic fatty liver disease. A decalogue from the Italian Association for the Study of the Liver (AISF) Expert Committee. Dig Liver Dis 2010;42:272-282.

21. Targher G, Bertolini L, Padovani R, et al. Relations between carotid artery wall thickness and liver histology in subjects with nonalcoholic fatty liver disease. Diabetes Care 2006;29:13251330.

22. Karakurt F, Carlioglu A, Koktener A, et al. Relationship between cerebral arterial pulsatility and carotid intima media thickness in diabetic and non-diabetic patients with non-alcoholic fatty liver disease. J Endocrinol Invest 2009;32:63-68.

23. Ying I, Saposnik G, Vermeulen MJ, Leung A, Ray JG. Nonalcoholic fatty liver disease and acute ischemic stroke. Epidemiology 2011;22:129-130.

24. Targher G, Day CP, Bonora E. Risk of cardiovascular disease in patients with nonalcoholic fatty liver disease. N Engl J Med 2010;363:1341-1350.

25. Thakur ML, Sharma S, Kumar A, et al. Nonalcoholic fatty liver disease is associated with subclinical atherosclerosis independent of obesity and metabolic syndrome in Asian Indians. Atherosclerosis 2012;223:507-511.

26. Assy N, Djibre A, Farah R, Grosovski M, Marmor A. Presence of coronary plaques in patients with nonalcoholic fatty liver disease. Radiology 2010;254:393-400.

27. Szollár L. Fatty liver and global cardiometabolic risk. Orv Hetil 2010;151:1946-1950.

28. Manousou P, Kalambokis G, Grillo F, et al. Serum ferritin is a discriminant marker for both fibrosis and inflammation in histologically proven non-alcoholic fatty liver disease patients. Liver Int 2011;31:730-739.

29. Marchesini G, Bugianesi E, Forlani G, et al. Nonalcoholic fatty liver, steatohepatitis, and the metabolic syndrome. Hepatology 2003;37:917-923.

30. Zelber-Sagi S, Nitzan-Kaluski D, Halpern Z, Oren R. NAFLD and hyperinsulinemia are major determinants of serum ferritin levels. J Hepatol 2007;46:700-707.

31. Pang JH, Jiang MJ, Chen YL, et al. Increased ferritin gene expression in atherosclerotic lesions. J Clin Invest 1996;97: 2204-2212.

32. Brea A, Mosquera D, Martín E, Arizti A, Cordero JL, Ros E. Nonalcoholic fatty liver disease is associated with carotid atherosclerosis: a case-control study. Arterioscler Thromb Vasc Biol 2005;25:1045-1050.

33. Fracanzani AL, Burdick L, Raselli S, et al. Carotid artery intima-media thickness in nonalcoholic fatty liver disease. Am J Med 2008;121:72-78.

34. Kim HC, Kim DJ, Huh KB. Association between nonalcoholic fatty liver disease and carotid intima-media thickness according to the presence of metabolic syndrome. Atherosclerosis 2009; 204:521-525.

35. Nambi V, Chambless L, He M, et al. Common carotid artery intima-media thickness is as good as carotid intima-media thickness of all carotid artery segments in improving prediction of coronary heart disease risk in the Atherosclerosis Risk in Communities (ARIC) study. Eur Heart J 2012;33:183-190. 